\title{
EL ALUMNADO CON ALTAS CAPACIDADES: LIDERAZGO, EDUCACIÓN INCLUSIVA Y TALENTO
}

\section{STUDENTS WITH HIGH INTELLECTUAL CAPACITIES: LEARDERSHIP, INCLUSIVE EDUCATION AND TALENT}

Antonio Carlos González López'1 : IES San Isidoro de Cartagena, Murcia. España antoniocarlos.gonzalez@murciaeduca.es

\section{RESUMEN}

El alumnado con Altas Capacidades escolarizado en nuestros centros educativos parece poseer una capacidad de liderazgo y talento para la organización humana y el trabajo cooperativo. Por ello, se hace necesario un acercamiento conceptual sobre liderazgo y talento en la educación inclusiva de este alumnado con Altas Capacidades. En este artículo, se analizarán diferentes puntos de vista actuales, y concluiremos, que el liderazgo puede darse en cualquier persona humana con una inteligencia normal. Por último, plantearemos que todo centro educativo debe ser una institución que favorezca cualquier tipo de talento del alumnado.

PALABRAS CLAVE: Altas Capacidades, Talento, Liderazgo, Inteligencia, Educación Especial, Educación Inclusiva.

\section{ABSTRACT}

Students with High Intellectual Capacities enrolled in our schools seem to possess leadership skills and talent for human organization and teamwork. Therefore, a conceptual approach on leadership and talent in the inclusive education of students with High Capacities becomes necessary. In this article, different current views will be discussed, and we conclude that leadership can occur in any human person with normal intelligence. Finally, we conclude that every school must be an institution that encourages any student talent.

KEY WORDS: High Intellectual Capacities, Talent, Leadership, Intelligence, Special Education, Inclusive Education.

\footnotetext{
${ }^{1}$ Antonio Carlos González López: IES San Isidoro, Cartagena, España. Investigador Externo del Grupo Desarrollo Personal. Universidad Católica de San Antonio de Murcia. Campus los Jerónimos s/n. Murcia. España.
} 


\section{Cómo citar el artículo:}

Antonio Carlos González López. (2013) EL ALUMNADO CON ALTAS CAPACIDADES: LIDERAZGO, EDUCACIÓN INCLUSIVA Y TALENTO. Revista de Ciencias de la Comunicación e Información. (Diciembre 2013). Año XXVII (30), 1-24 ISSN: 0213-070-X. Recuperado: http://www.revistaccinformacion.net/ARTICULOS/2013\%20e\%20Bea/2013\%20e-

\%20E|\%20alumnado\%20con\%20altas\%20capacidades.\%20Gonz\%C3\%A1lez\%20L\%C3\% B3pez.pdf

\section{INTRODUCCIÓN}

Vivimos tiempos de interés por la diversidad, la innovación y la calidad educativa. (Torre, Oliver y Sevillano, 2008). Especialmente ha crecido el interés por la respuesta educativa al alumnado con Altas Capacidades, y ligado a ello, los conceptos de liderazgo y talento que si bien en un principio, han estado unidos al mundo de la economía y la empresa, han sido trasladados al contexto de los sistemas educativos. Este planteamiento ha de ir dirigido a la mejora de las necesidades educativas de este tipo de alumnado, con el objetivo y convencimiento, de potenciar su desarrollo personal y social, y por supuesto, el bienestar común de la sociedad en la que se integra.

Fundamentalmente, el debate e interés por el alumnado con necesidades educativas especiales, se ha centrado en el alumnado con discapacidad, no obstante, la diversidad de las aulas nos ha llevado a fijarnos en aquel alumnado más "aventajado", que denominamos "superdotados", "talentos" y/o "genios". Con el término conceptual de alumnado con Altas Capacidades, utilizado actualmente, abarcamos una acepción más amplia de la clasificación de este alumnado. (Goleman, 2013; Marina, 2013).

La educación de calidad implementada en la diversidad conlleva la normalización y un modelo educativo inclusivo, donde este alumnado con Altas Capacidades o Talentos, deben educarse en los centros ordinarios. Algunas voces autorizadas, argumentando que esa respuesta retrasa la educación de los alumnos más dotados, proponen su escolarización en centros específicos para alumnado con Altas Capacidades. Bien es verdad, que la simpatía entre pedagogos y psicólogos a este tipo de educación son menores y las tendencias actuales, abogan por la inclusión del alumnado en grupos heterogéneos, eso sí, con programas específicos que ayuden al avance educativo que sus posibilidades presentan. (Jiménez Fernández, 2010).

\section{OBJETIVOS}

Los principales objetivos de esta comunicación se basan en una revisión actualizada de la conceptualización de Liderazgo, Talento y Altas Capacidades, dentro del contexto escolar, es decir, dentro de modelos educativos institucionalizados, abiertos, inclusivos y democráticos. Nos basaremos en diversos autores y teorías 
sobre el Liderazgo, y estableceremos consideraciones y estrategias de mejora para la innovación y la calidad de la respuesta educativa con este alumnado.

Hemos utilizado una metodología descriptiva de análisis de diferentes documentos legislativos, educativos y entrevistas, dentro de un marco escolar propio, como el diagnóstico por parte del Departamento de Orientación, para encontrar a este tipo de alumnado.

\section{METODOLOGÍA}

\subsection{Conceptualización de Liderazgo, Talento y Altas Capacidades}

Dentro de este marco no ha sido fácil para los investigadores, definir al alumno "aventajado intelectualmente" o "superdotado". (Jiménez Fernández, 2010). En el campo de la literatura de la psicopedagogía, se han utilizado diferentes denominaciones para el alumno con alta capacidad, que han supuesto conceptos sinónimos como: "superdotado", "sobredotado" y "alumno de alta capacidad".

Torrego, Boal, Bueno y otros (2011, p.13) define "superdotado" como aquella persona que tiene un "rendimiento por encima de lo normal...".

En primer lugar, daremos una definición de "Líder o Liderazgo". M.C. Medina Domínguez (2013, p.43), entiende como líder o liderazgo: “...la capacidad que tiene una persona para conseguir que los demás hagan lo que tienen que hacer con una actitud de voluntariedad y compromiso, sacando lo mejor de ellos mismos en un clima de crecimiento y equilibrio emocional".

En segundo lugar, "Talento" (termino lat. talentum: moneda antigua de los griegos), se puede definir como "la aptitud natural para hacer una cosa, entendimiento o inteligencia". (Lorenzo, 2006, p.73)

Según ambas definiciones, el talento aplicado a las relaciones humanas produce "liderazgo".

Actualmente, se usan diferentes vocablos sinónimos de Talento como son: genio, precocidad, prodigio, superdotado, superdotación, talento desaprovechado y talento discapacitado (Feldhusen, 1987, Benito, 1994, 1992; Soriano, 1994; Lorenzo y Martínez, 1995 a, b; 1996 a, b, c; 1998 a, b; 1999, 2000, 2002, 2003 a, b, c, d, e, f, 2004; citado en Lorenzo, 2006, p.74; Jiménez Fernández, 2010, pp. 28-29). Y más, recientemente, se utiliza el término "Altas Capacidades". (Jiménez Fernández, pp. 35-36)

Desde el punto de vista pedagógico, las definiciones tienen su origen en las teorías de las inteligencias múltiples (Gardner, 1983,1985) y del rendimiento (Renzulli, 1977). Para estas teorías, los alumnos "talentosos" se definen como "...alumnos que muestran habilidades específicas en áreas muy concretas. Se puede hablar de talento académico, talento matemático, talento verbal, talento motriz, talento social, talento artístico, talento musical, talento creativo..." (Torrego, Boal, Bueno y otros, 2011, p.14). 
La LOCME nos manifiesta que: “...todos y cada uno de los alumnos y alumnas serán objeto de una atención, en la búsqueda de desarrollo del talento, que convierta la educación en el principal instrumento de la movilidad social...”. (LOMCE, Preámbulo I, 2013:975858). Es decir, la potenciación del desarrollo del talento que cada individuo posee innato, será el instrumento del progreso y la movilidad social del individuo en España.

Por último se atribuye el término de "Alta Capacidad", a aquellos sujetos que: ... presentan un nivel de rendimiento intelectual superior en una amplia gama de capacidades y aprenden con facilidad cualquier área o materia. Las diferencias son fundamentalmente cualitativas, es decir, presentan un modo de funcionamiento diferente a la hora de enfrentarse y resolver una tarea. Hay autores que distinguen los casos de superdotación extrema y así hablan de "superdotación de primer orden" (sujetos con productividad superior y $\mathrm{Cl}$ mayor de 155) y "segundo orden ( $\mathrm{Cl}$ entre 125 y 130). (Torrego, Boal, Bueno y otros 2013, pp.13-14).

Así pues, aunque existen numerosas definiciones sobre el alumnado "aventajado intelectualmente", nos postulamos por la acepción de alumnado con "Altas Capacidades Intelectuales" (Prieto, Sánchez López, y Garrido, 2009). En este sentido:

El concepto de altas capacidades que introduce la LOGSE, aunque no lo define, parece que ha sido bien acogido por ser un término más general que el de superdotación y reclama la atención sobre los talentosos, los niños/as precoces, y por qué no, sobre todo aquel alumnado que está demostrando diariamente que puede manifestar conductas propias del alumno bien dotado. También porque el punto fundamental, al hablar de altas capacidades es su carácter de potencialidad, frente a la exigencia de rendimiento recogida por otros/as conceptos y por la legislación educativa anterior. (Revista de Educación Inclusiva, nำ1, 2008, p.105, citado en Albes, Aretxaga, Etxebarria y otros, 2013.p.11).

\subsection{Modelos de Liderazgo}

En el contexto escolar el liderazgo ha ido unido a la gestión y organización de los centros educativos. Los últimos estudios para la mejora de la educación realizados por el "Australian Institute for Teaching and School Leardership" [Instituto australiano para Enseñanza y Escuela del Liderazgo] (2011, citado en Gros, Fernández Salinero, Martínez Martín y otros, 2013, p.5), establecen los modelos de liderazgo que deben tener efectividad en el contexto escolar: "Liderazgo pedagógico, liderazgo distributivo y liderazgo moral".

Procede destacar como el concepto de líder y liderazgo han sido conceptos que se asimilaban entre sí, en la línea de que según fuese el líder, así era el modelo de liderazgo. Además, las investigaciones de los últimos veinte años han llevado a una íntima relación de liderazgo con la inteligencia emocional (IE). (Goleman, 2013, Medina Domínguez, 2013) 
En una economía mundial basada en el trabajo del conocimiento y las comunicaciones, el valor del liderazgo se crea con el trabajo en equipo, que trasladamos al campo educativo.

En esta misma línea, aparece el concepto que Robert Sternberg y Wendy Willians de la Universidad de Yale. Ellos nos propusieron que nos debíamos fijar en el "Coeficiente intelectual colectivo", ya que la suma de todas las aptitudes da su máxima capacidad. No obstante, Daniel Goleman indica que: "...lo que determina la productividad real del colectivo no es su potencial teórico (es decir, su coeficiente intelectual colectivo), sino la forma de coordinar sus esfuerzos". (Goleman, 2013. p.13). En definitiva, el líder del equipo es quien debe coordinar los esfuerzos.

Por todo ello, no creemos que sea suficiente tener un nivel de inteligencia elevado, entendida como la capacidad de resolver problemas y elaborar soluciones útiles en situaciones problemáticas; el líder debe poseer la capacidad de entender lo que piensan los demás (empatía) y hacerse entender. Es decir, actuar de manera inteligente, manejando sus emociones y comprender las emociones de las personas que conforman el equipo de trabajo cooperativo o educativo. (Goleman, 2013)

En resumidas cuentas, Daniel Goleman, investiga cómo la inteligencia emocional correlaciona con la competencia de liderazgo. En su artículo "La inteligencia emocional en la Empresa" (1998) introdujo un marco que"... refleja cómo se traducen los principios básicos de la IE (es decir, la autoconciencia, la autogestión, la conciencia social y la capacidad de gestión de relaciones) en éxito laboral" (Goleman, 2013, p.12). Los estudios de Daniel Goleman revolucionaron el mundo empresarial, y esto sería trasladado al mundo de las Ciencias de la Educación, es decir, a la Organización y la Didáctica Escolar.

En otro orden de cosas, ya las Ciencias de la Educación habían realizado sus estudios en la clasificación de los diferentes "estilos de liderazgo", tanto en la formación del docente, los equipos directivos y el rol del alumnado dentro del aula, a lo largo del siglo XXI.

Con el objeto de contribuir a hacer efectivo las nuevas investigaciones. Daniel Goleman se cuestionó: ¿Qué hay que tener para ser líder? (Goleman, 2013.p.31).

El primer requerimiento que nos propone será que "los líderes más eficientes (...) poseen un gran nivel de lo que ha dado en llamarse inteligencia emocional" (Goleman, 2013 p.32), no olvidando los prerrequisitos de coeficiente intelectual y una buena educación.

A pesar de estos estilos, D. Goleman nos indica que: “...es mejor que un líder emplee un amplio repertorio de estilos. Los que dominan cuatro o más (en especial el autoritario, el democrático y el conciliador y el coach) logran el mejor clima laboral y el mejor rendimiento". (Goleman, 2013 pp.75-76).

No obstante, considerando la profundización en la acepción o definición de "Liderazgo", que nos comunican diferentes autores, recogemos un conjunto de definiciones: 
- El liderazgo es el comportamiento de un individuo (...) que dirige las actividades de un grupo hacia una meta común" (Hemphill y Coons, 1957, pág.7).

- El liderazgo es "el aumento de la influencia por encima del cumplimiento mecánico de las directrices habituales de la organización (Katz y Khan, 1978, pág.528).

- El liderazgo se ejerce cuando las personas (...) movilizan (...) recursos institucionales, políticos y psicológicos, entre otros para despertar, captar y satisfacer las motivaciones de sus seguidores (Burns, 1978, pág.18).

- El liderazgo se realiza en el proceso, mediante el cual uno o más individuos consiguen delimitar o definir la realidad de otros (Smirch y Morgan, 1992, pág.258)

- El liderazgo es el proceso de influir sobre las actividades de un grupo organizado hacia la consecución de sus metas (Raucht Behling, 1984, pág.46).

- El liderazgo se refiere a la articulación de las visiones, la incorporación de los valores y la creación de un entorno en el que se consiguen las cosas (Richar y Engle, 1986, pág. 206).

- El liderazgo es el proceso de insuflar un propósito (una dirección con sentido) al esfuerzo colectivo y de generar un esfuerzo voluntario para la consecución de los objetivos (Jacobs y Jaques, 1990, pág.281).

- El liderazgo es la capacidad de trascender una cultura (...) para iniciar procesos de cambio evolutivo más adaptativos (Schein, 1992, pág.2).

- El liderazgo es el proceso de hacer comprensibles las actividades de los individuos para ayudarlos a comprender y comprometerse (Drath y Palus, 1994, pág.4).

- El liderazgo es la capacidad de un individuo de influir sobre todos, motivarlos y facilitar su contribución a la eficacia y el éxito de la organización (House et al., 1999, pág. 184). (Yulk, 2008, p.3, citados en Medina Domínguez, 2013, p.42)

\subsection{El alumno de Altas capacidades: Modelos de clasificación}

Se ha recorrido un largo camino para la clasificación del alumnado "aventajado" o de "alta habilidad" en el marco de la Pedagogía y Psicología. Como característica general y común a todos los modelos la clasificación del alumnado de Altas Capacidades ha tenido una intencionalidad educativa.

La situación histórica nos exige la mención de los diferentes modelos: a) la teoría de Renzulli (1977), basada en el rendimiento intelectual; b) el modelo de la inteligencia triárquica de Stenberg (1985), que se basa en la explicación de los mecanismos de la inteligencia y las habilidades de los alumnos/as que la usan; c) el modelo de la inteligencia emocional de Gardner (1983,1985), nos permitirá comprender el término talento; d) y el último, que usamos en nuestro entorno educativo, se centra en el modelo de Castelló y Batllé $(1988,1998)$, que se centra en un modelo que evalúa la 
alta habilidad, basándose en juegos cognitivos, socio-afectivos y creativos. (Prieto, Sánchez López, y Garrido Gil,2009).

Sobre la base de las ideas expuestas nos centraremos en tres modelos:

\subsubsection{El Modelo de los Tres Anillos de Joseph Renzulli (1977)}

El modelo americano más reconocido ha sido el creado por el Dr. Joseph Renzulli, del Instituto de Investigación para la educación de los alumnos Superdotados (Research Instute for Gifted Education, University of Connecticut, USA). En el "Modelo de los Tres Anillos", el superdotado debe poseer para ser considerado como tal, la combinación de elementos propios de un buen sistema de tratamiento de información (inteligencia elevada), con una alta originalidad y pensamiento divergente (creatividad) y la motivación suficiente para garantizar la materialización de su potencial (implicación en la tarea). "El denominado modelo de la puerta giratoria y del triple enriquecimiento (...), y que más recientemente su autor ha denominado como el modelo de enriquecimiento escolar en sentido amplio" (Jiménez Fernández, 2010, p.8).

Los docentes que trabajan con alumnado de Altas Capacidades proponen el mayor peso del pronóstico basado en la habilidad intelectual y el alto rendimiento escolar. No obstante, las personas no tienen por qué tener un coeficiente intelectual alto, para ser muy productivas o talentosas en algunas áreas de su vida cotidiana.

Este tipo de alumnado "Talentosos o Superdotados" suelen ser originales e ingeniosos, más bien, creativos, aunque no siempre ha habido acuerdo acerca de cómo medir la creatividad. En el ámbito docente se ha medido por los trabajos del alumnado: experimentos científicos, juegos, expresión escrita, mapas, etc.). La dificultad de medir la creatividad ha dado lugar a poner en duda unos resultados poco objetivos.

Una característica que podemos destacar del alumnado aventajado intelectualmente" es su perseverancia en las tareas a resolver, es decir, dedican su energía para la resolución de tareas o vicisitudes complicadas (Goleman, 2013).

$Y$ podemos concluir, con la representación del modelo concebido como el resultado de tres variables: capacidad intelectual, motivación y creatividad. (Figura 1) (Jiménez Fernández, 2010, p.8). 


\section{Figura 1}

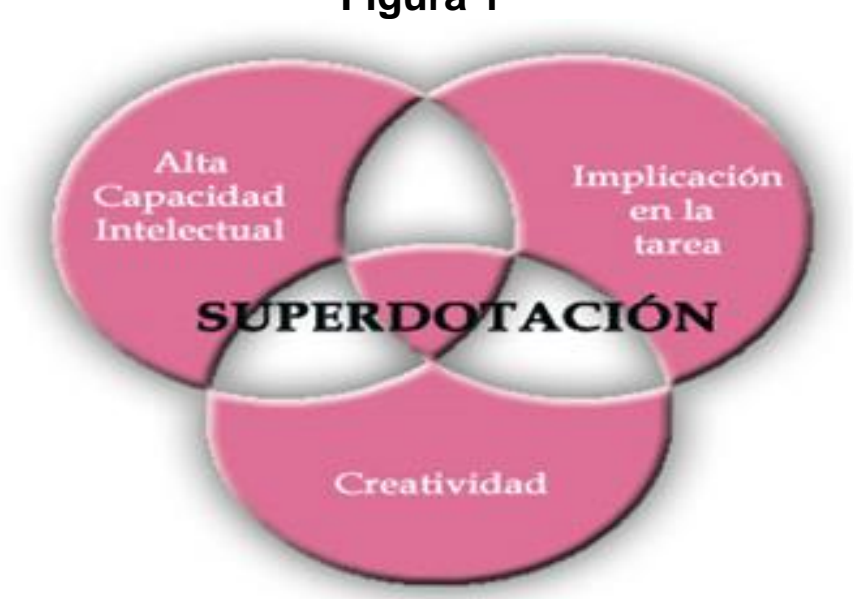

Fuente: Representación de la "Teoría de los Tres Anillos". Recuperado el día 21 de noviembre 2012, en http://www.psicoactiva.com/infantil/superdotado.htm

\subsubsection{El modelo de las Inteligencias Múltiples Howards Gardner (1983)}

Este autor ideó la "Teoría de las Inteligencias Múltiples", que iba más allá que considerar el rendimiento escolar. Esta teoría se basaba en las siguientes premisas:

- La inteligencia no es una capacidad unitaria, sino un conjunto de capacidades, talentos o habilidades cognitivas a las que denomina inteligencias. Según H. Gardner, la inteligencia es la capacidad para resolver problemas y crear productos que puedan ser valorados dentro de uno más ámbitos culturales.

- Cada una de estas inteligencias constituyen un sistema propio o independiente del resto.

- Para H. Gardner las inteligencias interactúan entre sí, de forma que un sujeto puede exhibirlas en un momento determinado, pero esta situación es fluida y cambiante. ( Jiménez Fernández, 2010).

H. Gardner no admite el modelo basado en la inteligencia de forma jerárquica y unitaria, especialmente en los tests de $\mathrm{Cl}$. El modelo de este autor, ha tomado fuerza en el ámbito docente y universitario. Aunque, esta teoría también presenta unos puntos fuertes y débiles dentro del marco de la pedagogía y psicología.

Para Howard Gardner, la inteligencia se basa en siete capacidades cognitivas o inteligencias humanas que son:
a) La inteligencia lingüística;
b) la lógica-matemática;
c) la viso-espacial;
d) la musical;
e) la físico-kinéstésica;
f) la interpersonal;
g) y la intrapersonal. (Figura 2) (Jiménez Fernández, 2010, p.13) 


\section{Figura 2}

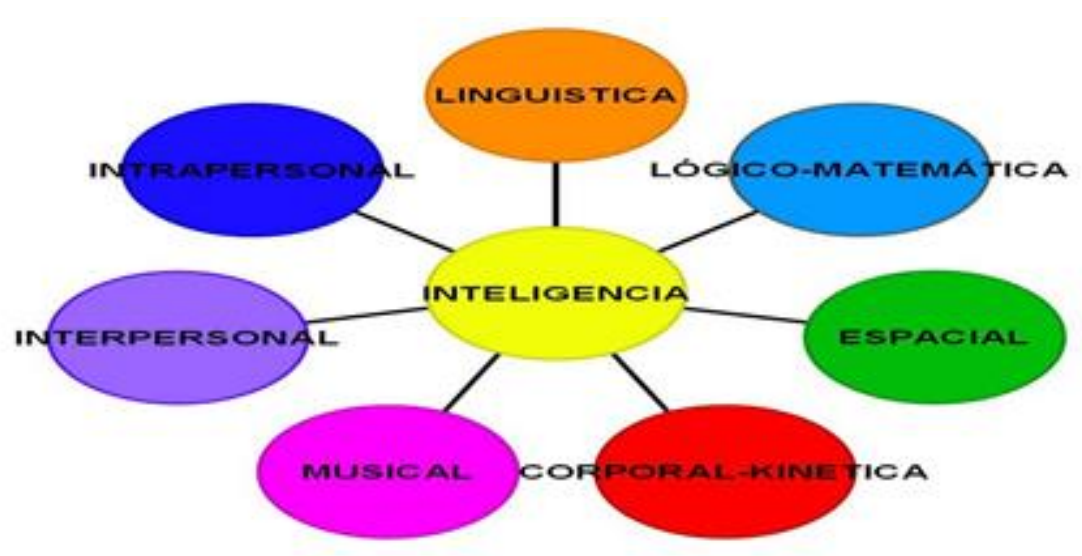

Fuente: Teoría de las Inteligencias Múltiples de H. Gardner. Recuperado el día 27 de noviembre de 2012, en: http://biogestion.blogspot.com.es/2006 1101 archive.html

\subsubsection{El Modelo de Castelló $(1986,1998)$}

En el año 1986 Castelló (Castelló, 1995) propuso operativizar el objeto de estudio de la superdotación, basándose en unos criterios para definir al superdotado:

- Dependencia de contexto y necesidades sociales.

- Importancia de la producción.

- Ponderación de factores implicados.

- Diferenciación cualitativa entre superdotado y talentoso en cuanto a características internas y potencial de producción.

- Capacidad de mediación de las características.

Nos encontrábamos con dos grupos de individuos. El primero, los superdotados, que son los excepcionales intelectualmente, ya que tienen un conjunto de características que garantizan un alto nivel de funcionamiento de toda la estructura del intelecto. $Y$ el segundo, los talentosos propiamente dicho, cuya característica fundamental es que tiene rendimientos parciales extraordinarios, en los factores mencionados por Castelló u otras áreas no estrictamente intelectuales.

En el año 1998, Castelló y Batllé proponen un protocolo de identificación basado en dos instrumentos de medida: BADyG (Batería de Aptitudes Diferenciadas y Generales, C. Yuste Hernanz, 1989), y el Test de pensamiento creativo de Torrance (Torrance Test of Creative Thinking, TTCT, 1974).

En realidad presentan una clasificación diferenciada de la alta habilidad en: superdotación, talento académico, talento figurativo, talento artístico figurativo, talento verbal, talento numérico, talento espacial y talento creativo. Es decir, intentan cubrir los problemas que existen frecuentemente con la terminología conceptual de uso referidos al alumnado "aventajado" (superdotación, talento, precocidad, etc.).

La concreción del modelo, se desarrolla en la Comunidad Autónoma de la Región de Murcia, bajo la supervisión del Equipo de Altas Capacidades de la Consejería de Educación, Universidades y Empleo y la colaboración de la Universidad de Murcia. 
En este desarrollo el modelo de diagnóstico y tratamiento educativo confluyen en un todo integrado. (Ferrándiz, Rojo y Ferrando, 2009).

\subsubsection{Diagnóstico}

Los padres o docentes son los primeros que detectan al alumnado de "alta habilidad". Posteriormente, se someten a una serie de pruebas objetivas para su diagnóstico correcto que son:

\section{En infantil y primaria:}

- BADyG Batería de Aptitudes Diferenciales y Generales.

- TTCT Test de Pensamiento Creativo de Torrance.

- Cuestionario para la Evaluación del Autoconcepto (Piers Harris)

- Batería de Socialización F. Silva Moreno y M. S. C. Martorell Pallas (BAS 1 y 2) dirigida a profesores y a padres.

- Adaptación del cuestionario de Inteligencia Emocional de Reuven Barón.

\section{En secundaria:}

- Test de Aptitudes Diferenciales (DAT-5) (versión 5 nivel 19).

- Batería de Aptitudes Generales y Diferenciales BADYG-M (subprueba de memoria oral).

- Tercer subtest del Test de Pensamiento Creativo de Torrance (TTCT), versión figurativa.

- La escala BFQ-NA de Claudio Barbaranelli, Gian Vittorio Caprara y Annarita Rabasca en su adaptación española V. Del Barrio Gándara, M.A. Carrasco Ortiz y F.P. Holgado Tello. Otras pruebas personalidad alternativas.

- El EQ-i: YV (Inventario de Inteligencia Emocional para niños y adolescentes, Bar-On y Parker, 2000).

- EQ-i: YV-O (Bar-On y Parker, en prensa), para valorar la inteligencia emocional de los alumnos, destinado a padres y profesores.

El modelo de Castelló y Batlle (1998) clasifica el alumnado de "alta habilidad" en superdotados y talentosos en función de los percentiles que los alumnos obtienen y que se plasman en la siguiente tabla (Tabla 1):

Tabla 1

\begin{tabular}{|c|c|c|c|c|c|c|c|}
\hline Aptitudes & SPD & TA & TAr & TM & TV & TL & TC \\
\hline Creatividad & 75 & & 80 & & & & 95 \\
\hline Lógica & 75 & 80 & & & & 95 & \\
\hline $\begin{array}{c}\text { Gestión } \\
\text { perceptiva }\end{array}$ & 75 & & 80 & & & & \\
\hline $\begin{array}{c}\text { Gestión } \\
\text { memoria }\end{array}$ & 75 & 80 & & & & & \\
\hline $\begin{array}{c}\text { Aptitud } \\
\text { verbal }\end{array}$ & 75 & 80 & & 95 & 95 & & \\
\hline $\begin{array}{c}\text { Aptitud } \\
\text { matemática }\end{array}$ & 75 & & & 95 & & \\
\hline
\end{tabular}




\section{\begin{tabular}{|c|l|l|l|l|l|l|l|}
$\begin{array}{c}\text { Aptitud } \\
\text { espacial }\end{array}$ & $\mathbf{7 5}$ & & $\mathbf{8 0}$ & & & & \\
\hline
\end{tabular}}

Fuente: Clasificación del alumnado de Altas Capacidades Intelectuales en: Superdotados y Talentosos, con indicación del valor del centil mínimo para cada una de las aptitudes. (SPD=Superdotados. TA= Talento académico. TAr=Talento artístico. TM=Talento matemático. TV= Talento verbal. TL=Talento Lógico. TC=Talento Creativo.)(Elaboración propia, citada en Castelló, 1995, pp.19-26)

De esta forma Castelló y Batlle (1998) establecen una clasificación del alumnado de Altas Capacidades en:

- Superdotado Cuando se obtienen percentiles iguales o superiores a 75 en lógica, verbal, matemático, gestión de memoria, creatividad y espacial.

- Talento Múltiple Configuración intelectual con altos recursos (Percentil 95) en varias aptitudes específicas (verbal, matemático, espacial...)

- Talentos complejos:

- Académico: Percentil 80 o más en aptitudes que configuran el talento lógico, verbal y la gestión de memoria.

- Artístico-figurativo: Percentil 80 o más en aptitudes que configuran el talento lógico, creativo y espacial.

- Figurativo: Percentil 80 o más en aptitudes lógicas y espaciales.

- Talentos conglomerados formados por la combinación de alguno de los talentos complejos con uno simple o con uno múltiple.

- Talentos simples:

- Espacial: Percentil igual o superior a 95 en aptitud espacial.

- Lógico: Percentil igual o superior a 95 en aptitudes lógicas.

- Verbal: Percentil igual o superior a 95 en aptitudes verbales.

- Creativo: Percentil igual o superior a 95 en creatividad.

Cada talento se evalúa en función de una o varias subpruebas en la batería BADyG para la etapa de infantil y cada uno de los ciclos de primaria ${ }^{2}$.

\subsubsection{Tratamiento educativo a la diversidad}

La respuesta educativa que se da al alumnado con Altas Capacidades se basa en:

- Flexibilidad en las actividades de clase con predominio de actividades de ampliación.

- La individualización de las actividades con el trabajo cooperativo que conllevan el desarrollo cognitivo, creativo, liderazgo, motivación y social.

- La identificación de la diferenciación del alumno con Altas Capacidades lleva a la "Diferenciación Curricular" por medio de: actividades de ampliación curricular, enriquecimiento curricular en el aula y enriquecimiento curricular extraescolar en el centro educativo o institución de educación superior.

\subsection{La educación inclusiva para el alumnado con Altas Capacidades}

La Educación Inclusiva ha supuesto un modelo de interpretación conceptual de la superación de la integración escolar.

\footnotetext{
${ }^{2}$ Protocolo de evaluación psicopedagógica para alumnado de Altas Capacidades en E. Infantil, E. Primaria y E. Secundaria. Seminario Regional de Altas Capacidades. CARM.
}

Revista de Ciencias de la Comunicación e Información. XXVII, 30. 1-24 (2013) 
Stainback y StainbacK (1995, citado en Sola, López Urquízar y Cáceres, 2009 p.36) plantean un modelo educativo o paradigma actual, en el que todo el alumnado en una escuela gratuita, currículo común y sistema abierto, está incluido totalmente.

La "Educación Inclusiva" es un sistema pedagógico que nos planteamos para el alumnado con Altas Capacidades. El objeto de dar respuesta a la diversidad de este alumnado dentro y/o fuera del aula, con apoyos específicos para desarrollar su individualidad como "alumnado aventajado intelectualmente". Hay unos hechos que justifican la Educación Inclusiva en el alumnado de "alta habilidad", y es que este tipo de alumnado suele presentar problemas de relación social y/o habilidad social, en algunas ocasiones.

Además, Caldevilla indica que: "Las relaciones humanas están perdiendo su esencia" (2010, p.127). Esto obliga al profesor, a disponer de unas actividades, estrategias y medidas que ayuden ante cualquier situación difícil del alumnado con Altas Capacidades o niños/as simplemente. Para que "sus relaciones humanas sean más normalizadas". Con el fin de facilitar su "desarrollo personal", proporcionándoles unos valores "éticos-humanos" propios del siglo XXI.

Ahora bien, según, el modelo pedagógico que postulaba, Susan Bray Staninback (2001, citado en Pujols, 2011, p.18), independientemente que sean alumnos/as con Altas Capacidades, todos son alumnos dentro del aula y presentan unas necesidades propias, que deben ser tomadas en consideración. A este respecto la Didáctica, Didáctica Especial y la Organización Escolar deben adaptarse al alumnado. El fin es permitir la participación activa dentro del aula con un currículo común para todos/as los alumnos/as, y unas adaptaciones si fuesen necesarias. (Palomares, 1998; Ainscow, 2001; Sola, López Urquízar y Cáceres, 2009; Medina, De la Herrán y Sánchez Romero, 2011).

Igualmente, abogamos por la línea de pensamiento de Stainback y Stainback (1990, p.5, citado en Sola, López Urquízar y Cáceres, 2009 p.36), que establecen tres razones fundamentales para implementar la Educación Inclusiva:

- el aprender a vivir y trabajar con iguales en su contexto natural y comunidad-

- evitar la exclusión social, motivados por los efectos de la segregación

- y por último, realizar lo que es justo y equitativo en educación.

Gordon Porter (2002, p.7, citado por Pujóls, 2011, p.18), nos indica muy acertadamente el derecho del alumnado a participar en la escuela común, con unas garantías de "normalidad", y si presentasen condición de discapacidad, con sus adaptaciones necesarias que den respuesta a sus necesidades educativas.

En suma, la definición de Educación Inclusiva nació con unas perspectivas que para Mariano Naradowski (2008, p.32) indicaban las fuertes desigualdades en materia de igualdad de oportunidades. Una vez aplicada se ha demostrado que se puede desvanecer la calidad de la educación ante el conjunto de problemas de acceso e inclusión y es que, pesar del esfuerzo, la cuestión de la "inclusión" conlleva un desafío difícil. Los programas educativos para la mejora de la educación han llevado a la dualidad "inclusión-exclusión", y para actuar con políticas inclusivas, Soto 
Calderón (2003, citado en Narodowski, 2008.p.33), nos indica como paso previo clarificador de la inclusión educativa: “...el análisis y la discusión de los diferentes procesos en que se ha enmarcado la experiencia educativa con necesidades educativas especiales..."., a lo que habría que añadir el estudio de los procesos de formación docente y las buenas prácticas educativas que confluyan en la implementación de este paradigma o modelo educativo.

A este respecto, se realizó una investigación sobre "Medidas y Estrategias de Educación Inclusiva: Alumnado con NEE de la ESO en las provincias de Albacete y Murcia” (González López, 2013). A través de los resultados obtenidos en las entrevistas realizadas, pudimos concluir que en lo referido al alumnado de Alta Capacidad, se daba prioridad al modelo inclusivo, ya que en relación a estos alumnos, los profesionales entrevistados respondieron que: “...deben ser tratados en Centros ordinarios..." (González López, 2013, p.263).

Al comparar estas evidencias, Mariano Naradowski (2008 p.35) define la Educación Inclusiva bajo el prisma de que incluir es "redistribuir" para compensar las desigualdades en el aspecto económico, y el "reconocer", en el aspecto cultural las diferencias personales.

Es más, nos referiremos a los postulados de la UNESCO $(1995,2011)$, contra la lucha de la "exclusión social", ratificando que: "La educación inclusiva y de calidad se basa en el derecho de todos los alumnos a recibir una educación de calidad que satisfaga sus necesidades básicas de aprendizaje y enriquezca sus vidas".

Para Gordon Porter (2002, p.7, citado por Pujóls, 2011, p.18), “...los estudiantes con necesidades especiales o discapacitados van a la escuela donde irían si no fuesen discapacitados y van a una clase normal, con los compañeros de su misma edad". Es decir, nos indica muy acertadamente el derecho del alumnado a participar en la escuela común, con unas garantías de "normalidad", y si presentarán condición de discapacidad, sus adaptaciones necesarias que den respuesta a sus necesidades educativas.

La Educación Inclusiva no significa una "educación igualitaria", no conlleva que el papel de "educar" sea asumido por el Estado. Es un modelo de "participación" basado en los tres ejes fundamentales de la comunidad educativa: familia, escuela y Estado.

La educación actual, retoma la necesidad de un reconocimiento a la diversidad de los integrantes de la educación, para que intentemos aparcar la denominación de diferentes, especiales o aventajados. De ahí, los cambios conceptuales de "Retraso Mental" por "Discapacidad intelectual" (Schalock, 2007) y "Niños prodigio" por "Altas Capacidades". La clasificación de éstos últimos, se fundamenta basándose en modelos que tienen como objeto de estudio las capacidades (Marland, 1972), el rendimiento (Renzulli, 1977), los aspectos cognitivos y los socioculturales. (Tannenbaum, 1986) (Mönks y Van Boxtel; Mönks, 1992) (Sánchez López, 2007).

En términos de educación actual, retomamos la unidad básica de la sociedad que es la familia. La familia es el componente básico de aprendizaje de los hijos/as y 
subsidiariamente el Estado. El alumnado de Altas Capacidades debe educarse en la diversidad. Los valores personales deben aprenderse dentro de la familia y un estado social, cuestión que autores agnósticos y de origen católico postulan. (Castell, 2007; Sarmiento y Escribá, 2009). La familia sigue siendo el primer agente socializador del alumnado, incluido el que tratamos de Altas Capacidades, ya que estos alumnos pueden presentar problemas de "relación social", que bajo el prisma de un desarrollo personal basado en valores y estructuración familiar de afectividad, se evitarían.

En definitiva, la familia es la célula básica de la sociedad. El alumnado de Altas Capacidades debe educarse en la diversidad pero con unos valores instruidos por la familia y el estado social. Autores de índole agnóstica así nos lo manifiestan (Castel, R., 2006³; Augusto Sarmiento y Javier Escrivá-Ivars, 2009²).

La iglesia, en su preocupación por lo social, no ha dudado en críticar aquellas ideologías cuyas políticas educativas o sociales, no promuevan el desarrollo integral del hombre (Juan Pablo II, 1987).

Nuestra propuesta, no sólo es recogida por sociólogos agnósticos, otros autores así lo manifiestan:

Para afrontar los desafíos sociales y económicos que se plantean en el mundo actual, se requiere la generosa aportación de todos. Los administradores públicos, a los que el pueblo ha confiado funciones de guía y gobierno, deben referirse constantemente a él, considerando la actividad política y administrativa como un servicio.

Por tanto, el hombre ha de ocupar el lugar central de todos vuestros proyectos e intervenciones. Prestad atención particular a la familia, cuyo papel es fundamental en la construcción de la sociedad. (Sarmiento y Escrivá-Ivars "Enchiridion Familia”, recogiendo las palabras de Juan Pablo Il en su discurso "Sono Lieto"5).

La familia como núcleo es el primer contexto de aprendizaje del alumnado. El modelo tradicional de familia, ha dejado de lado roles rígidos de hombre y mujer, y entre padres e hijos. Los padres han optado por una educación bajo el signo de la libertad, la comunicación, el diálogo y una mayor tolerancia. Sin embargo este estilo educativo mencionado parece que no tenido los resultados esperados, ya que ha dado lugar a niños más dependientes, conductas disruptivas en los centros, problemas de violencia, familias desestructuradas, etc.

\footnotetext{
${ }^{3}$ Entrevista a Robert Castel. Por Editores Topía. Publicado en Abril 2006. Recuperado el 3 de diciembre de 2013, en: http://www.topia.com.ar/articulos/entrevista-robert-castel

${ }^{4}$, loannis Pauli PP. II. Discurso "Sono Lieto", en la Audiencia a los Presidentes de las Regiones de las Provincias Autónomas de Italia, 29 noviembre 2001. (Sarmiento, A. y Escribá.Ivars, 2009, pp.85138514)

${ }^{5}$ Ioannis Pauli PP. II. Discurso "Me es gráto", al nuevo Embajador de España ante la Santa Sede, con ocasión de la presentación de las Cartas Credenciales, 18 de junio 2004((Sarmiento, A. y Escribá.Ivars, 2009, p.8707)
} 
Es importante volver a "educar a las familias" sobre el desarrollo psicológico, social y emocional de sus hijos/as, procurando la estimulación intelectual con un ambiente emocionalmente afectivo positivo, y en definitiva, fomentar la independencia, la autonomía y un proyecto de vida para sus hijos/as (Javayales, s.f.).

Un estilo educativo familiar apropiado, no sólo para el alumnado de Altas Capacidades sino para el alumno normal o discapacitado, debe conllevar estas premisas: a) la comunicación abuelos-padres-hijos; b) la exigencia de conductas y valores apropiados a cada edad, es decir, tareas/obligaciones dentro de la familia, ya que el cumplimiento de las normas -(aún con flexibilidad)-, les prepara para el futuro de su entorno social; c)y por último, el afectivo hacia los hijos, basándose en los valores humanos.(Javaloyes, s.f.) (Peña y otros., 2012, 2013).

La Educación Inclusiva no se contradice con los postulados del humanismo cristiano, ya que éstos están basados en una sociedad con los valores de justicia, dignidad del ser humano y/o la convivencia humana con ideales de libertad, justicia y solidaridad mundial. ${ }^{6}$

Partiendo de estos supuestos una Educación Inclusiva necesita la participación de la familia del alumnado, no sólo del centro educativo. Por otro lado el papel que se demanda del maestro es que sea fuerte, con autoridad y con libertad en el compromiso social de su tarea en sí (Narodowski, M., 2008).

En estas condiciones, el maestro no sólo educa, sino que se erige como dinamizador de los elementos nuevos de la enseñanza-aprendizaje. El alumnado del siglo XXI recibe información y formación con nuevos procesos de enseñanzaaprendizaje: accesibilidad a la red, MOOCS, móvil, Smarphone, Tablets, TICS, etc. (Caldevilla y González Oñate, 2011 Gea, Montes, Rojas y otros, 2013).

Para el "alumnado aventajado" nos posicionamos además, en una educación a través del trabajo cooperativo, (principio de la Educación Inclusiva). Esta estrategia didáctica ayuda a conseguir las metas de flexibilidad, individualidad, motivación, liderazgo e inclusión social. (Ainscow, 1995; Ainscow, 2001; Arnaiz, 2003; Echeita, 2008; Casanova, 2011; González López, 2013).

¿Cómo hacer para evitar que las políticas de discriminación positiva se transformen en lo contrario?

En este contexto, Echeita nos indica que:

...no pretendo caer en la ingenuidad de pensar que la educación escolar es la puerta más importante para paliar los procesos de exclusión social (...), pero es una de ellas, y nuestra responsabilidad como educadores es que lo que hagamos puertas adentro en la escuela sea parte de la solución y no parte del problema. (Castell R., 2004, pp.65-69, citado en Echeita, 2008 p.21).

¿Cómo generar una escuela inclusiva dentro de un marco escolar rígido o nuevo? 
La respuesta es sencilla mediante el "trabajo cooperativo" o "trabajo por proyectos", tanto docentes como discentes (Ferrándiz, Rojo y Ferrando, 2009). La motivación, el liderazgo y el trabajo responsable van unidos.

El "trabajo cooperativo" se define como:

...el uso didáctico de equipos reducidos de alumnos, generalmente de composición heterogénea en rendimiento y capacidad, aunque ocasionalmente pueden ser homogéneos, utilizando una estructura de la actividad tal que asegure la participación equitativa ( para todos los miembros del equipo tengan las mismas oportunidades de participar) y se potencie al máximo la interacción simultánea entre ellos, con la finalidad de que todos los miembros de un equipo aprendan contenidos escolares, cada uno hasta el máximo de sus posibilidades y aprenda, además, a trabajar en equipo. (Jhonson, Jhonson y Holubec, con las aportaciones de Spencer Kagan 1999, citado en Pujols, 2011, p.26).

¿Cómo podemos incidir en la organización escolar de los centros para llevar a cabo una educación inclusiva innovadora y de calidad?

No me cabe duda, cambiando la mentalidad del profesorado, la formación y considerar la educación como un reto humano: ¡Somos educadores!

¿Y la Educación Inclusiva, se refiere sólo al alumnado de Altas Capacidades?

Mi respuesta, rotunda:-¡No! La inclusión responde a todos/as las alumnos del sistema educativo, incluido los normales, los de necesidades educativas especiales, los problemas del aprendizaje, el alumnado multicultural y las altas capacidades. Las medidas que deben enmarcarse en un currículo común, abierto y flexible, deben incidir en las adaptaciones necesarias para que dentro de la individualidad se coopere dentro del grupo, consiguiendo un proyecto de futuro personal basado en valores humanos. (Ainscow, 1995; Ainscow, 2001; Arnaiz, 2003; Palomares y González López, 2012; González López, 2013; Peña y otros., 2012, 2013).

¿Quién o quiénes deben adaptarse a este paradigma de la Educación Inclusiva?

¡Toda la comunidad educativa!

¿Qué entiendo por comunidad educativa?

Se entiende: alumnos/as, padres y madres, profesores/as, personal no docente y la administración educativa.

Y por último: -¿Qué estrategias metodológicas puedo aplicar al alumnado con Altas Capacidades dentro del aula normal?:

Aprender junto a alumnos diferentes, partiendo del reconocimiento de las diferencias. (Oliver, 2003; De la Torre, Oliver, Sevillano y otros, 2008; Medina, De la Herrán, Sánchez Romero y otros, 2011) 


\subsection{Estrategias para desarrollar un liderazgo en el campo de la educación: docente y discente.}

La Didáctica, Didáctica Especial y la Organización Escolar deben adaptarse al alumnado, con el fin de conseguir una participación activa dentro del aula con un currículo común, abierto y unas adaptaciones necesarias. De esta manera nos planteamos la Educación Inclusiva como modelo o paradigma educativo para el alumnado con Altas Capacidades.(Palomares, 1998; Sola, López Urquízar y Cáceres, 2009).

Las ideas y reflexiones expresadas en apartados anteriores se resumen en:

Por ello para conseguir el pleno desarrollo de las capacidades, se requiere la colaboración de todos los componentes de la comunidad educativa, es decir, del esfuerzo compartido. Pero la responsabilidad del éxito escolar de todos los alumnos no solo recae sobre el individuo considerado sino también sobre sus familias, los profesores, centros docentes, las Administraciones educativas y, en última instancia, sobre la sociedad en su conjunto, responsable última de la calidad del sistema educativo. Cada uno de ellos tendrá que realizar una contribución específica. (Torrego, Boal, Bueno y otros, 2011 p.54).

Esta postura nos lleva a una autonomía, participación en los procesos y descentralizar el liderazgo del maestro/a tradicional. Implica el postulado de un trabajo más individualizado bajo el prima del "trabajo cooperativo" o "trabajo por proyectos" (Medina, De la Herrán, Sánchez Romero y otros, 2011, p.68) dentro del aula. Esta estrategia o sistema de enseñanza-aprendizaje disminuye las diferencias y reparte el trabajo basado en los talentos individuales o liderazgos innatos.

Ainscow corrobora esta idea: "Una escuela basada en una estructura cooperativa aprovechará probablemente los conocimientos técnicos de todo su personal" (Ainscow, 1995, p.37, citado en Sola, López Urquízar y Cáceres, 2009, p.396).

En la LOE (2006), se establecían dos tipos de medidas, "medidas ordinarias" y/o "medidas extraordinarias". (González López, 2013, p.149).

Las medidas ordinarias tenían lugar en el proceso de normalización, es decir, dentro de las medidas que se adoptan en el currículo sin llegar a las excepcionales, si resultarán necesarias. Todo esto, conllevaba unas medidas de Atención a la Diversidad en los centros educativos o institutos, con una actuación global, que implicaba a toda la comunidad educativa. Estas medidas se especificaban en las programaciones de aula, Equipo Docente, Departamentos, Claustro y/o Centro Educativo. Estas medidas, se subdividían en dos clases: adaptaciones curriculares de ampliación o enriquecimiento curricular. Éstas tendrán lugar en el aula con el/los alumnos concretos y mediante actividades y estrategias metodológicas interdisciplinares (Jiménez Fernández, 2010) que podrían ser del tipo (Figura 3): 
Figura 3

\section{METODOLOGÍA-ACTIVIDADES POR ÁREAS RECOMENDADAS}

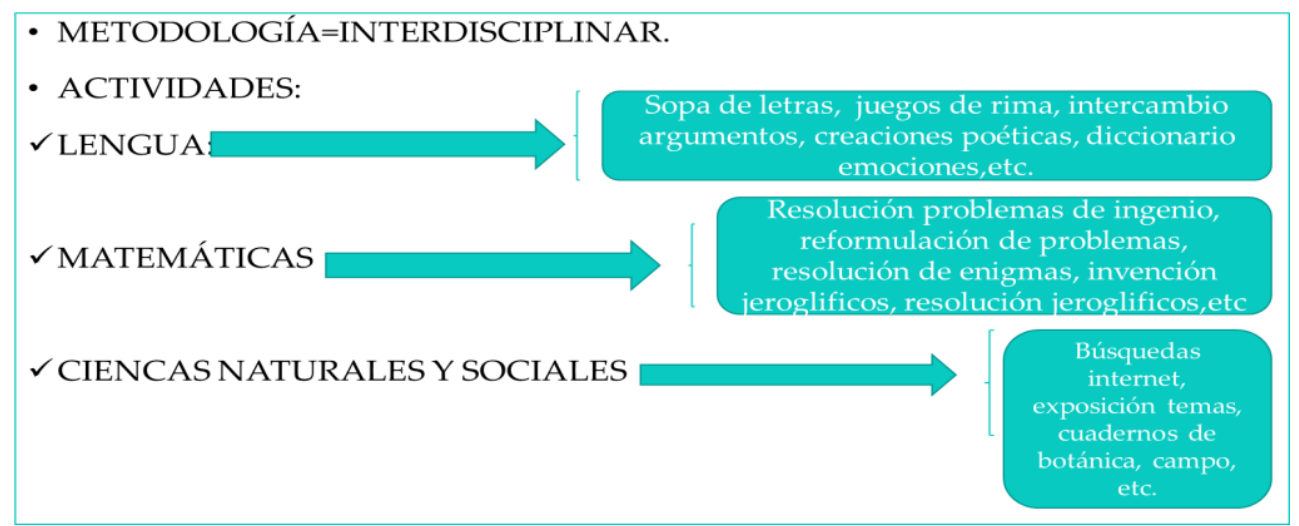

Fuente: Estrategias y Medidas de carácter inclusivo para el alumnado con Altas Capacidades (Elaboración propia, citado en Torrego, Boal, Buevo y otros, 2011 pp.58-60)

En la Comunidad de la Región de Murcia, el diagnóstico y la respuesta educativa se basó en los modelos de Castelló y Batllé (1998). Las medidas educativas se clasificaron en medidas ordinarias y específicas. Las ordinarias se plasmaban en las programaciones equipos docentes, los departamentos de área y/o didácticos de los institutos o colegios. Mientras que, las medidas específicas se alternaron con talleres de enriquecimiento curricular, con temáticas variadas cada año, dando oportunidad para el desarrollo del alumno de Altas Capacidades a su ritmo, interés, capacidad y estilo cognitivo propio.

\section{RESULTADOS}

El centro donde me sitúo es un centro; IES. San Isidoro, de un barrio periférico suburbano (Los Dolores) en la ciudad de Cartagena. El instituto tiene estudios de la ESO y Bachillerato. El centro perseguía como objetivo principal, la continuidad y mejora, de la puesta en práctica del proyecto como, "Centro Preferente de Escolarización de Alumnos/as con Altas Capacidades”. Estos cambios eran de sobra conocidos por el profesorado, ya que se habían acogido en el curso 2009/2010. Durante el curso 2010/11, se continúa con la implementación del proyecto, cuyos resultados fueron significativos, ya que se encontró, alumnado con talento 0 habilidad alta, que pasaba desapercibido. Los pasos del proyecto llevaba consigo: identificación; diagnóstico; análisis por la "Comisión de Coordinación”; propuestas de actividades y desarrollo de las actividades por los departamentos, tanto de ampliación, como de enriquecimiento curricular ofertadas por la Consejería de Educación de la Comunidad Autónoma de la Región de Murcia. El organigrama de funcionamiento del proyecto del "Centro de Acogida de Alumnos con Altas Capacidades" se estructuró en: 
- Cargos unipersonales:

- Coordinador proyecto de altas capacidades.

- Orientador.

- Coordinador proyecto ARCE.

- Coordinadora proyecto Comenius.

- Coordinadora Bachillerato Investigación.

- Comisión de coordinación del proyecto.

- Coordinador.

- Orientador.

- Directora.

- Profesor de Pedagogía Terapéutica.

El tamaño de la muestra de alumnado sobre la que se trabajo fue de 609 alumnos/as. Además, el centro contaba con la cantidad 69 profesores/as. En este apartado, referente a los resultados me centraré, los resultados que obtuvimos referentes al diagnóstico, basándonos en las pruebas psicométricas, que propio programa de la Consejería de Educación propone, anteriormente expuesto en otro apartado. ¿Qué busco con la plasmación de estos resultados? La evidencia que ha llevado a la administración a poner estos programas, ya que este tipo de alumnado con Altas Capacidades, pasaba desapercibido, necesitando una respuesta educativa adecuada. Los resultados tras el diagnóstico por parte del Departamento de Orientación, en coordinación con los órganos unipersonales y la comisión de coordinación fueron los siguientes:

\section{Tabla 2}

\begin{tabular}{|l|r|r|r|r|r|}
\hline $\begin{array}{c}\text { Alumnado } \\
\text { diagnosticado para } \\
\begin{array}{c}\text { Altas Capacidades } \\
\text { IES San Isidoro curso } \\
\mathbf{2 0 1 1 / 1 2}\end{array}\end{array}$ & frec. & $\begin{array}{c}\text { frec. } \\
\text { relativa }\end{array}$ & \% & $\begin{array}{r}\text { rec. abs. } \\
\text { acum. }\end{array}$ & $\begin{array}{r}\text { frec. rel. } \\
\text { acum. }\end{array}$ \\
\hline Talentos simples & 6 & 0,01 & 1 & 6 & 0,01 \\
\hline Talentos múltiples & 4 & 0,01 & 1 & 10 & 0,02 \\
\hline $\begin{array}{l}\text { Talentos } \\
\text { conglomerados }\end{array}$ & 12 & 0,02 & 2 & 22 & 0,04 \\
\hline Superdotados & 6 & 0,01 & 1 & 28 & 0,05 \\
\hline Total muestra & 608 & & & & \\
\hline
\end{tabular}

Fuente: Elaboración propia,( citado en Aniorte, A.,2011) 


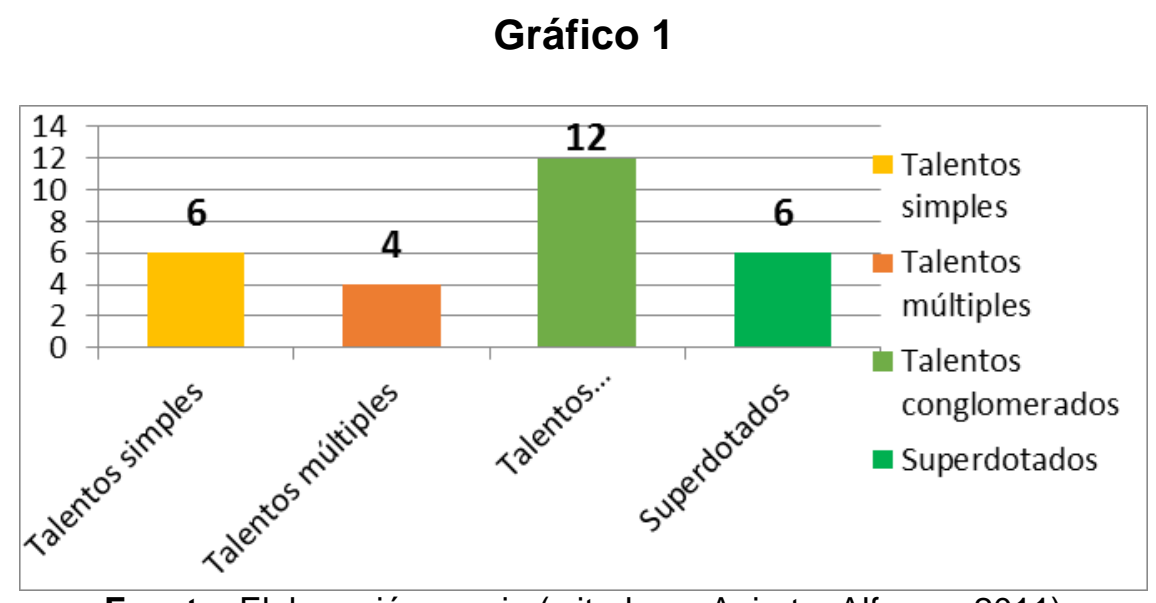

Fuente: Elaboración propia,( citado en Aniorte, Alfonso ,2011)

En conclusión, la elaboración del proyecto de centro para la identificación; diagnóstico; análisis y propuestas de actividades para el alumnado con Altas Capacidades. Así pues, supuso un reto de búsqueda de una acción científicatecnológica. Además, investigar los elementos de armonía e identidad relevantes y creativas, que puedan identificar a los líderes. Esto llevó, a conseguir una optimización de los aprendizajes esperados de este alumnado. Es decir, un conocimiento práctico de un saber teórico. En definitiva, la identidad de los líderes y el alumnado con Altas Capacidades. Por último, quedó demostrado que algunos/as alumnas/as de estas características diversas pasaban desapercibidos para la comunidad educativa.

\section{CONCLUSIONES}

Para concluir, discernimos una serie de conclusiones que brevemente serían:

El talento se puede desarrollar por medio de la educación y la enseñanzaaprendizaje. (Mazuela y Urcelay, 2003; Marina, 2013)

El liderazgo es innato en alumno aventajado pero cualquier persona lo puede alcanzar.( Mazuela y Urcelay, 2003; Mellim, 2011; Goleman, 2013; Marina, 2013; Medina Domínguez, 2013)

El término Altas Capacidades se acuña para dar un acercamiento conceptual más amplio del alumnado con estas características.( Torrega, Boal, Bueno y otros, 2011)

Las investigaciones en el campo de la inteligencia emocional ha derivado al estudio de las emociones y el liderazgo en el campo educativo. El líder debe tener la cualidad de emocionar al equipo para aunar esfuerzos de trabajo cooperativo.( De la Torre, Oliver, y Sevillano, 2008; Melim, 2011)

En la Comunidad de la Región de Murcia se implantó el Protocolo de Castell y Batllé $(1988,1998)$.

La inclusión es un modelo educativo válido para normalizar al alumnado con "alta habilidad".( Ainscow, 1995; Ainscow, 2001; Arnaiz, 2003; Sola, López Urquízar y Cáceres, 2009); Casanova, 2011; Albes, Aretxaga, Etxebarria y otros, 2013) 
Las investigaciones de las "Buenas Prácticas Educativas" han puesto en evidencia el término inclusión, haciendo necesario su redefinición y compromiso participativo de la comunidad educativa. (Narodowski,2008; Casanova, 2011)

El proceso de construcción de un proyecto de centro da una validez al marco teórico y reflexivo de una comunidad educativa.(Medina, A, De la Herrán, A., Sánchez, C. y otros (2011)

La familia es el núcleo principal del aprendizaje del alumno, motor de la sociedad.(UNESCO, 1995,2005,2011)

El maestro debe tener un papel fuerte y dinamizador en el modelo inclusivo. Nuestra actuación será transmisores y receptores de la información, narradores, evaluadores, consejeros y compañeros en las actividades de enseñanza-aprendizaje. (Ainscow, 1995; Ainscow, 2001; Arnaiz, 2003; Casanova,2011; Palomares y González López, 2012; González López, 2013) La Iglesia por medio de su compromiso social, educativo y de la familia. Promueve el desarrollo integral del hombre. La libertad de enseñanza y conciencia no se contrapone a una Educación Inclusiva, sino que se promueve desde un humanismo cristiano. Así como, desde otras perspectivas educativas. ( UNESCO, 1995; 2005 ,2011; Juan Pablo II,2001,2004; Oliver, 2003; Echeita, 2008; Peña y otros., 2012.2013; Marina, 2013)

Y por último, cualquier ser humano puede llegar a ser un líder en la aptitud o capacidad que sea educado o motivado. (Mazuela y Urcelay, 2003;Medina Domínguez, 2013; Goleman, 2013)

\section{REFERENCIAS}

\section{Libros:}

Ainscow, M. (1995). Necesidades especiales en el aula. Guía para la formación del profesorado. Unesco-Narcea: Madrid.

Ainscow, M. (2001). Desarrollo de las escuelas inclusivas. Narcea:Madrid.

Arnaiz, P. (2003). Educación inclusiva: una escuela para todos. Editorial Aljibe: Archidona (Málaga).

Albes, C., Aretxaga, L., Etxebarria, I. y otros. (2013). Orientaciones educativas. Alumnado con altas capacidades intelectuales. $1^{\text {a }}$ Edición. Gobierno Vasco. Departamento de Educación, Política Lingüística y Cultura. Servicio de Publicaciones del Gobierno Vasco: Victoria.

Casanova, Ma A. (2011). Educación inclusiva: un modelo de futuro. Wolters Kluwer España: Madrid.

De la Torre, S, Oliver, C. y Sevillano MáL. y otros (2008). Estrategias didácticas en el aula. Buscando la calidad y la innovación. Primera edición. Aula Abierta. UNED: Madrid.

Gardner, H. (2006). Multiple Intelligences: New Horizons by Basic Books. 
Goleman D. (2013). Liderazgo. El poder de la inteligencia Emocional. 1a Edición. Traducido por Carlos Mayor. Grupo ZETA. Ediciones B.S.B.: Barcelona.

González López, A.C. (2013). Medidas y Estrategias de Educación Inclusiva: Alumnado con NEE de la ESO en las provincias de Albacete y Murcia. Tesis doctoral inédita. Ediciones Universidad de Granada: Granada.

Jiménez Fernández, C. (2010). Diagnóstico y educación de los más capaces. Segunda edición. Pearson: Madrid.

Ley Orgánica, General del Sistema Educativo, de 3 de octubre de 1990 (BOE de 4 de octubre). (LOGSE) (LOE).

Ley Orgánica, 2/2006, de 3 de mayo (BOE de 4 de mayo), de Educación.

Mazuela, A. y Urcelay, J. (2003). Liderazgo y dirección de personas. UNED: Madrid.

Marina, J.A. (2013). Talento, Motivación e inteligencia. Las claves de una buena educación. 1a Edición. Editorial Planeta: Barcelona.

Medina Domínguez, Maㅗ. C. (2013). Formación de Líderes en Inteligencia Emocional y Gestión del Talento.1aEdición. Editorial Universitas: Madrid.

Medina Rivilla, A., De la Herrán, A., Sánchez Romero (coords.) y otros (2011). Formación Pedagógica y práctica del profesorado. UNED. Editorial Universitaria Ramón Areces: Madrid.

Melim Soares, G. I. (2011). Emociones, personalidad y liderazgo: un estudio con directores de centros educativos.Tesis doctoral inédita. Universidad de Cádiz: Cádiz.

Oliver $\mathrm{M}^{\mathrm{a}} \mathrm{C}$. (2003). Estrategias didácticas y organizativas ante la diversidad. Ediciones OCTAEDRO-EUB: Barcelona.

Palomares, A. (1998). Educación Especial y Atención a la Diversidad. L. Universidad Albacete: Albacete.

Peña B. (coord.) y otros. (2012). Desarrollo Humano.1ํㅡㄹ Edición. UCAM. Visión Libros: Murcia.

Peña, B. (coord.) (2013). Human Development. Yking books. PublishersExporters: Jaipur.

Sánchez López, C. (2007). Configuración cognitivo-emocional en alumnos de altas habilidades. Tesis doctoral inédita. Departamento de Métodos de Investigación y Diagnóstico en Educación. Universidad de Murcia: Murcia. 


\section{EL ALUMNADO CON ALTAS CAPACIDADES: LIDERAZGO, EDUCACIÓN INCLUSIVA Y TALENTO}

Sarmiento, A. y Escrivá-Ivars, J. (2009). Enchiridion Familiae. Textos del Magisterio Pontificio y Conciliar sobre el Matrimonio y la Familia. (Siglos I a XXI). XI Juan Pablo II 82001-2005). Instituto de Ciencias para la Familia. Universidad de Navarra.

Sola Martínez, T., López Urquizar, N. y Cáceres Reche, M로.( 2009). La educación especial en su enmarque didáctico y organizativo. Grupo Editorial Universitario: Granada.

Torrego, J.C. (Coord.), Boal M. ${ }^{\mathrm{a}}$ T., Bueno, A. y otros (2011). Alumnos con Altas Capacidades y Aprendizaje Cooperativo. Un modelo de respuesta educativa. Edición Fundación SM: Madrid.

Artículos en revistas:

Caldevilla, D. (2010)." El hombre binario". Comunicación y hombre: revista interdisciplinar de la comunicación y humanidades. Noviembre, 2010, Núm.6. Dedicado a: Estrategias persuasivas en la comunicación. pp.125-138

Castelló, A. (1995)."Configuración cognitiva de algunos superdotados y algunos talentos". Aula de Innovación Educativa [Versión electrónica]. Revista aula de Innovación Educativa №45. pp.19-26.

Echeita, G. (2008). "Inclusión y Exclusión Educativa"." Voz y Quebranto. Revista Latinoamericana de Educación Inclusiva. Septiembre 2008/Núm.2. Edita Escuela Diferencial. Facultad de Ciencias de la Educación. Universidad Central de Chile: Santiago de Chile. pp.19-29.

Lorenzo García, R. (2006).” ¿A qué se le denomina talento? Estado del arte acerca de su conceptualización". Revista Intangible Capital, Enero- Marzo de 2006, №11, Vol.2, págs.77-163.

Narodowski, M. (2008). "La Inclusión educativa. Reflexiones y propuestas entre las teorías, las demandas y los slongans". Voz y Quebranto. Revista Latinoamericana de Educación Inclusiva.

Palomares A. y González López, A. C. (2012). "La educación inclusiva en el alumnado con necesidades educativas especiales en la Educación Secundaria Obligatoria de la Región de Murcia". Vol.48, Núm.2 pp.267-283

Porter, G.L. (2001). "Elements crítics per a escoles inclusive. Creant l'escola inclusiva: una perspectiva canadenca basada en quinze anys d'experiència. Suports. Revista Catalana d'Educació Especial i Atenció a la Diversitat. Vol.5, núm.1, pp.6-14

Shalock, R., Luckasson, R. y otros (2007): "El nuevo concepto de retraso mental: comprendiendo el cambio al término discapacidad intelectual". Traducido por Laura E. Gómez Sánchez. . Revista Española sobre Discapacidad Intelectual. Vol.38 (4), Núm. 224. Universidad de Salamanca (INICO). pp. 5-20. 
UNESCO (1995). Conferencia Mundial sobre las necesidades especiales.Ministerio de Educación y Ciencia: Salamanca.

$\underline{\text { Recursos electrónicos: }}$

Aniorte, A.(2011). Ponencia: "Proyecto de centro: atención educativa a alumnos con altas Capacidades". IES San Isidoro. Cartagena. Consultado el 3 de enero de 2013.

Disponible http://diversidad.murciaeduca.es/altascapacidades/viijornadas.php

en:

Caldevilla, D. y González Oñate, C. (2011). "Herramienta Educativa "MOS y las TIC. Técnica en pro de la Innovación Educativa." Etic@net. Año 2011, Núm. 10 (Junio). Dedicado a: la Educación Multimedia. Consultado 3 de enero de 2013. Disponible en:

http://dialnet.unirioja.es/servlet/articulo?codigo=3702703

"Aprendizaje por proyectos". NorthWest Regional Educational Laboratory.Junta de Andalucía. Consultado el día 15 de diciembre de 2012. Disponible en: ectos.pdf

www.juntadeandalucia.es/averroes/ escuelatic20/didactica/aprendizaje x proy

Entrevista de actualidad a "Roberts Castell" en julio de 2009/ Por Ángels Doñate. "Hoy sentimos que hemos perdido el dominio sobre nuestro porvenir social". Universitat Oberta de Catalunya. Consultado el día 15 de diciembre de 2012. Disponible en:

http://www.uoc.edu/portal/es/sala-depremsa/actualitat/entrevistes/2009/robert castel.html

Ferrándiz, C., Rojo A. y Ferrando M. (2009). "Intervención Educativa en alumnado con Altas Capacidades Intelectuales". Unidad 9. Temas de Diversidad. Gómez Portillo, A. (Coord.) y otros. Centros de Profesores de Cieza. Universidad de Murcia. Consejería de Educación, Ciencia e Investigación de Murcia. Consultado el día 8 de diciembre de 2012.Disponible en:

http://teleformacion.carm.es/moodle/mod/resource/view.php?id=86764

Gea M., Montes, R., Rojas; B. y otros (2013). "Formación abierta sobre modelos de enseñanza masivos: nuevas tendencias hacia el aprendizaje social". Equipo docente de Tecnologías Digitales Internet y Aprendizaje 2.0) Curso de formación abierta de la UGR). Centro de Enseñanzas Virtuales de la Universidad de Granada. Consultado el día 3 de enero de 2013. Disponible en:

https://lsi.ugr.es/ rosana/investigacion/files/abiertaUGR CAFVIF2013.pdf

Ionees Paulus PP. (1987). Sollicitudoo rei sociales. Carta encíclica del Papa Juan Pablo II, promulgada el 30 de diciembre de 1987, con ocasión del vigésimo aniversario de la "Encíclica Populorum Progresio", acerca de la preocupación social de la Iglesia. Consultado el día 15 de diciembre de 2012. Disponible en:

www.vatican.va/holy father/john paul ii/encyclicals/documents/hf ipii enc 30121987 sollicitudo-rei-socialis sp.html 


\section{EL ALUMNADO CON ALTAS CAPACIDADES: LIDERAZGO, EDUCACIÓN INCLUSIVA Y TALENTO}

Javaloyes, A. (s.f.). "El papel de la familia en la educación de los hijos". Doctora en Psiquiatría. Asociación de Pediatría Extrahospitalaria de la Provincia de Alicante. Recuperado el día 15 de diciembre de 2012, en:

www.apepa.org/index.php?menu=documentos\&id=2\&id doc=304\&show=1

MECD (2013). "Borrador del Proyecto de real decreto por el que se establece el currículo básico de la Educación Primaria, de la Educación Secundaria Obligatoria y del Bachillerato" (10/12/13). Recuperado el día 20 de diciembre de 2012, en:

www.mecd.gob.es/servicios-al-ciudadano-mecd/dms/mecd/servicios-alciudadano-mecd/participacion-publica/curriculo-basico/RD-curr-culo-LOMCE-PRESO-BACH-V1-10-12-13.pdf

Ponencia Ángel Guirado Sánchez. Doctor en psicología y especialista en altas capacidades. Formador y director del Observatorio sobre Superdotación y Talentos de la UNED. (2012). "Las propuestas de intervención educativa desde los modelos representacionales de los niños con altas capacidades y de sus educadores". III Jornadas de Altas Capacidades Intelectuales: Intervenciones Educativas. 20 de octubre. COSMOCAIXA-Barcelona. Consultado el día 9 de diciembre de 2012. Disponible en:

www.uned.es/cagirona/cat/superdotacio/Resumen Ponencia III Jornadas AC. $\underline{\mathrm{pdf}}$

Prieto, M.D., Sánchez López, M.C., y Garrido Gil, C.F (2009). "Características del alumnado con Altas Capacidades". Unidad 8. Temas de Diversidad. Gómez Portillo, A. (Coord.) y otros. Centros de Profesores de Cieza. Universidad de Murcia. Consejería de Educación, Ciencia e Investigación de Murcia. Consultado el día 7 de diciembre de 2012. Disponible en:

http://teleformacion.carm.es/moodle/mod/resource/view.php?id=86763

Principales modelos de clasificación de superdotación y talentosos. Consultado el día 7 de diciembre de 2012. Disponible en:

www.tdx.cat/bitstream/handle/10803/10993/SanchezLopez04de12.pdf;jsessionid=B4 46E79E2F5D341CDF127816BADE5762.tdx2? sequence $=4$

UNESCO (2005). Informe de Seguimiento de la Educación para Todos en el Mundo 2005: El imperativo de la calidad. Consultado el día 20 de diciembre de 2012. Disponible en:

www.unesco.org/new/es/education/themes/leading-the-internationalagenda/efareport/reports/2005-quality/

UNESCO (2011). Educación Especial e Inclusión Educativa. Estrategias para el desarrollo de escuelas y aulas inclusivas. VI Jornadas de Cooperación Educativa con Iberoamérica sobre Educación Especial e Inclusión Educativa. La Antigua, Guatemala, 5 al 9 de octubre de 2009. Ponencia Marco: "Aprendizaje cooperativo y educación inclusiva: una forma práctica para que puedan aprender juntos diferentes" de Peré Pujolàs Maset. Universidad de Vic. (Barcelona). Proyecto auspiciado por el Ministerio de Educación de España. Publicado por OREAL/UNESCO: Santiago de Chile. Consultado el día 15 de diciembre de 2012. Disponible en: http://unesdoc.unesco.org/images/0019/001931/193130s.pdf 
UNESCO. "Lucha contra la exclusión". Definición de Educación Inclusiva. Consultado el día 15 de diciembre de 2012, disponible en:

www.unesco.org/new/es/education/themes/strengthening-education-

systems/inclusive-education/ 